\title{
Síndrome metabólica em adolescentes de diferentes estados nutricionais
}

\author{
Metabolic syndrome in adolescents of different nutritional status
}

Antonio Stabelini Neto', Rodrigo Bozza ${ }^{2}$, Anderson Ulbrich², Luis Paulo Gomes Mascarenhas ${ }^{3}$, Margaret Cristina da Silva Boguszewski ${ }^{4}$, Wagner de Campos $^{5}$

\section{RESUMO}

'Centro de Ciências da Saúde, Universidade Estadual do Norte do Paraná (UENP), Jacarezinho, PR, Brasil

2 Programa de Pós-Graduação em Educação Física, Universidade Federal do Paraná (UFPR) Curitiba, PR, Brasil

${ }^{3}$ Programa de Pós-Graduação em Saúde do Adolescente, UFPR, Curitiba, PR, Brasil ${ }^{4}$ Departamento de Educação Física, UFPR, Curitiba, PR, Brasil ${ }^{5}$ Departamento de Pediatria, UFPR, Curitiba, PR, Brasil

Objetivo: Verificar a prevalência de síndrome metabólica (SM) em adolescentes de diferentes estados nutricionais. Sujeitos e métodos: A amostra constitui-se de 582 adolescentes de 12 a 18 anos de idade. A classificação do estado nutricional por meio do índice de massa corporal (IMC) foi realizada utilizando as tabelas de referência do NCHS. A SM foi diagnosticada se três ou mais fatores de risco estivessem presentes. Resultados: A prevalência geral de SM foi 6,7\% (IC: 4,9\%-9\%); rapazes 9,4\%; moças 4,1\%. Prevalência de SM de $17,2 \%$ (IC: $10 \%-28,2 \%$ ) e $37,1 \%$ (IC: $23,2-53,7 \%$ ) foi observada em adolescentes com sobrepeso e obesidade, respectivamente. Todos os adolescentes obesos tinham pelo menos um fator de risco presente e demonstraram elevada razão de prevalência de SM comparados aos adolescentes com peso normal (RP: 11,1; IC: 5,75-21,47). Conclusão: Alta prevalência de SM foi observada em adolescentes obesos, neste sentido, estratégias de prevenção devem ser concentradas no controle do peso corporal desde o início da adolescência. Arq Bras Endocrinol Metab. 2012;56(2):104-9

\section{Descritores}

Síndrome metabólica; adolescentes; obesidade

\begin{abstract}
Objective: To investigate the prevalence of metabolic syndrome (MetS) in adolescents of different nutritional status. Subjects and methods: The sample consisted of 582 adolescents aged 12 to 18 years. Body mass index (BMI) classification of nutritional status was performed using the NCHS growth charts. MetS diagnosis was determined by the presence of three or more risk factors. Results: Overall MetS prevalence was 6.7\% (Cl: $4.9 \%-9 \%)$; in boys, prevalence was 9.4\%; and in girls, $4.1 \%$. MetS prevalence was $17.2 \%(\mathrm{Cl}: 10 \%-28.2 \%)$ and $37.1 \%(\mathrm{Cl}: 23.2 \%$ $53.7 \%$ ) in overweight and obese adolescents, respectively. All obese adolescents had at least one risk factor present, and demonstrated high MetS prevalence ratio compared with adolescents of normal weight (PR: 11.1; $\mathrm{Cl}$ : 5.75-21.47). Conclusion: High prevalence of MetS was observed in obese adolescents. Prevention strategies should focus on body weight control since the beginning of adolescence. Arq Bras Endocrinol Metab. 2012;56(2):104-9
\end{abstract}

Correspondência para: Antonio Stabelini Neto Universidade Estadual do Norte do Paraná, Campus Jacarezinho Centro de Ciências da Saúde Alameda Padre Magno, 841 86400-000 - Jacarezinho, PR, Brasil asneto@uenp.edu.br

Recebido em 26/Jan/2011 Aceito em 6/Fev/2012

Keywords

Metabolic syndrome; adolescents; obesity

\section{INTRODUÇÃO}

A síndrome metabólica (SM) se refere a uma agregação de fatores de risco cardiovascular representada por hipertensão, obesidade abdominal, hipertrigliceridemia, baixa concentração de lipoproteínas de alta densidade (HDL-C) e intolerância à glicose. O diagnóstico de SM é estabelecido quando três ou mais dos elementos coexistem no mesmo sujeito (1). A SM está associada com um risco elevado de desenvolvimento de doença arterial coronariana (2) e diabetes melito (3). 
Estimativas contemporâneas demonstram que, nos Estados Unidos, a SM afeta $22 \%$ de sua população adulta e $4,2 \%$ dos adolescentes entre 12 e 16 anos de idade (4). No Brasil, a prevalência da SM encontrada recentemente entre adultos foi de $21,6 \%$ (5) e 29,8\% (6), no entanto, ainda são escassos estudos sobre a prevalência da SM em crianças e adolescentes brasileiros.

Estudos têm sugerido que a etiologia da SM em indivíduos jovens está ligada ao excesso de peso corporal $(7,8)$. Consequentemente, como a prevalência de obesidade infantil aumentou nos últimos dez anos (9), é esperado que o número de jovens com diagnóstico de SM também tenha aumentado.

Um dos problemas relacionados à falta de informações sobre as taxas de prevalência de SM em brasileiros jovens é que ainda grande parte dos estudos nacionais produzidos nessa área se concentra nos fatores de risco biológicos separadamente. Assim, devido à escassez de estudos sobre a prevalência de SM na população pediátrica, o objetivo deste estudo foi verificar a prevalência de SM em adolescentes de 12 a 18 anos de diferentes estados nutricionais.

\section{MÉTODOS}

\section{Sujeitos}

Dados dos projetos "Fatores de risco para aterosclerose relacionados ao nível de aptidão cardiorrespiratória em adolescentes", "Impacto do tamanho ao nascimento e do estilo de vida sobre os fatores de risco metabólico e cardiovascular em crianças e adolescentes" e "Aptidão física, estilo de vida e síndrome metabólica em adolescentes" foram utilizados para desenvolvimento da pesquisa. Apenas os estudantes entre 12 e 18 anos de idade matriculados na rede pública de ensino do estado do Paraná foram incluídos nas análises. Os critérios de exclusão foram a presença conhecida de diabetes e uso de medicamentos que alteram a pressão arterial, a glicose ou o metabolismo lipídico.

Os bancos de dados continham informações de 601 adolescentes de 12 a 18 anos de idade, 239 adolescentes da cidade de Curitiba, 249 da cidade de São Mateus do Sul e 113 da cidade de Jacarezinho, sendo os dados coletados entre os anos de 2006-2009. Ao final da análise, 19 sujeitos foram excluídos das análises por apresentarem dados incompletos.

O termo de consentimento livre e esclarecido informado foi obtido a partir dos pais ou responsável legal.
Essas pesquisas foram aprovadas pelos Comitês de Ética da Universidade Federal do Paraná e Universidade Estadual do Norte do Paraná (Resolução 196/96).

\section{Procedimentos}

Para determinar a estatura, foi utilizado um estadiômetro vertical portátil da marca WCS com escala de $0,1 \mathrm{~cm}$. Para definir a massa corporal, utilizou-se uma balança digital portátil marca PLENNA com resolução de $100 \mathrm{~g}$. $\mathrm{O}$ avaliado deveria estar descalço e vestindo somente trajes leves. O índice de massa corporal (IMC) foi calculado pela equação: [massa corporal $(\mathrm{kg}) /$ estatura $(\mathrm{m})^{2}$ ].

Para a classificação do estado nutricional de acordo com os percentis do IMC, foram utilizados os seguintes pontos de corte: baixo peso: IMC $<$ percentil $5^{\circ}$; normal: IMC $\geq$ percentil $5^{\circ}$ e IMC $<$ percentil $85^{\circ}$; sobrepeso: IMC $\geq$ percentil $85^{\circ}$ e $<$ percentil $95^{\circ}$; obesidade: IMC $\geq$ percentil $95^{\circ}$. Os sujeitos foram classificados utilizando as tabelas de referência desenvolvidas de acordo com o sexo e a faixa etária pelo National Center for Health Statistics (10) para indivíduos de 2 a 20 anos de idade.

A circunferência da cintura foi mensurada no ponto médio entre o último arco costal e a crista ilíaca. A pressão arterial foi mensurada por meio do método auscultatório, seguindo os parâmetros estabelecidos na literatura (11). A pressão arterial sistólica (PAS) e diastólica (PAD) foram aferidas no braço direito do avaliado, utilizando um esfigmomanômetro com capacidade de $300 \mathrm{mmHg}$ e variação de $2 \mathrm{mmHg}$. A PAS foi definida como o som de Korotkoff fase l e a PAD como o som de Korotkoff fase 5. A mensuração era realizada após o indivíduo permanecer sentado em repouso por um período de 5 minutos, com as costas apoiadas, os pés no chão e o braço direito apoiado com a fossa cubital ao nível do coração. Duas leituras foram realizadas com intervalo de 10 minutos entre as medições, sendo considerado o valor médio entre as duas mensurações. Caso as medidas diferissem em mais que $2 \mathrm{mmHg}$, o protocolo era repetido.

Foram coletados aproximadamente $8 \mathrm{~mL}$ de sangue de cada indivíduo para análise laboratorial e determinação do HDL-C, triglicérides e glicemia. Os sujeitos foram instruídos com antecedência sobre os cuidados que deveriam tomar para participarem da coleta sanguínea: a) jejum prévio obrigatório de no mínimo $10 \mathrm{~h}, \mathrm{~b}$ ) evitar o consumo de álcool três dias antes do teste; c) evitar o abuso alimentar (em especial gordura) no dia anterior ao teste. 
A retirada de sangue foi realizada por enfermeiras do próprio laboratório onde as amostras sanguíneas foram analisadas, no período das 7 às 9 horas da manhã. As amostras foram processadas e analisadas no mesmo dia da coleta, sendo utilizado o soro para as análises. Foi usado o método enzimático-colorimétrico automatizado.

\section{Definição de síndrome metabólica}

Foi utilizada a proposta de Cook e cols. (4), que adaptaram os critérios para adultos do III NCEP-ATP e propuseram como diagnóstico de SM em adolescentes a presença no mesmo sujeito de três ou mais dos seguintes componentes: PAS e/ou $\mathrm{PAD} \geq$ percentil $90^{\circ}$ para idade, sexo e percentil da estatura (11); triglicerídeos $\geq 110 \mathrm{mg} / \mathrm{dL}$; HDL-C $\leq 40 \mathrm{mg} / \mathrm{dL}$; glicemia de jejum $\geq 110 \mathrm{mg} / \mathrm{dL}$; obesidade abdominal por meio da circunferência da cintura $\geq$ percentil $90^{\circ}$ para idade e sexo. Como não existem valores referenciais de circunferência da cintura para crianças e adolescentes, foi utilizada na presente pesquisa a classificação de todos os adolescentes de acordo com a idade e o sexo em percentis de circunferência da cintura da própria amostra.

\section{Estatística}

A prevalência da SM e a agregação de seus componentes foram determinadas para os diferentes estados nutricionais por meio da distribuição de frequência com seus respectivos intervalos de confiança para 95\%. Testes de qui-quadrado foram calculados para comparar as prevalências de SM entre os estratos. Razão de prevalência e intervalos de confiança de $95 \%$ foram calculados para associar o estado nutricional com o diagnóstico de SM entre indivíduos com excesso de peso comparados aos adolescentes com peso normal. As análises estatísticas foram realizadas utilizando o programa SPSS, versão 15.0 para Windows (Chicago, IL, EUA), com um nível de significância estipulado em $\mathrm{p}<0,05$.

\section{RESULTADOS}

As características da amostra são apresentadas na tabela 1 . Considerando a amostra total, 6,7\% dos adolescentes foram diagnosticados com SM, sendo esta prevalência mais elevada no sexo masculino $(9,4 \%)$ do que no feminino $(4,1 \%)$.
$\mathrm{Na}$ observação do estado nutricional, $5,2 \%$ dos escolares apresentavam baixo peso (masculino $5,4 \%$; feminino: 4,6\%), $11 \%$ apresentaram sobrepeso (masculino: 11,7\%; feminino: 9,9\%) e 6\% eram obesos (masculino: 6\%; feminino: 6\%).

A proporção de sujeitos com 1 ou mais componentes da SM inadequados de acordo com as proposições da literatura adotados pelo presente estudo é apresentada na tabela 2. Verificou-se que mais da metade da amostra apresenta um ou mais fatores de risco, com maior prevalência entre os jovens do sexo masculino.

Quando avaliamos a agregação dos fatores de risco para SM de acordo com o estado nutricional, notamos que nenhum dos indivíduos de baixo peso foi diagnosticado com SM. A contraposto, todos os obesos apresentavam ao menos um componente da SM.

Entre os fatores de risco analisados isoladamente, os que apresentaram um maior número de casos entre os adolescentes avaliados foram as concentrações de HDL-C abaixo de $40 \mathrm{mg} / \mathrm{dL}$, acometendo 29,2\% dos indivíduos, seguidas da pressão arterial aumentada $(18,9 \%)$, hipertrigliceridemia $(18,3 \%)$ e hiperglicemia $(4,7 \%)$.

Levando em conta a associação do estado nutricional com diagnóstico de SM, os escolares com sobrepeso apresentaram uma razão de prevalência de 5,1 (IC: 2,47-10,70) e os obesos, de 11,1 (IC: 5,75-21,47) comparados aos seus pares com peso esperado, demonstrando que o excesso de peso corporal aumenta consideravelmente a chance de diagnóstico de SM.

Tabela 1. Prevalência de SM em adolescentes de 12 a 18 anos estratificados pelo sexo, grupo etário e estado nutricional

\begin{tabular}{lccc}
\hline & $\begin{array}{c}\text { Número de } \\
\text { sujeitos }\end{array}$ & $\begin{array}{c}\text { Percentual de sujeitos } \\
\text { com SM (IC 95\%) }\end{array}$ & p \\
\hline Total & 582 & $6,7 \%(4,9-9,0)$ & \\
$\begin{array}{l}\text { Sexo } \\
\quad \text { Masculino }\end{array}$ & 287 & $9,4 \%(6,5-13,3)$ & 0,01 \\
$\quad$ Feminino & 295 & $4,1 \%(2,3-7,0)$ & \\
Idade & & & \\
12-14 anos & 380 & $6,6 \%(4,5-9,5)$ & 0,872 \\
15-18 anos & 202 & $6,9 \%(4,2-11,3)$ & \\
Estado nutricional & & & 0,000 \\
$\quad$ Baixo peso & 30 & 0 & \\
Normal & 453 & $3,3 \%(2,0-5,4)$ & \\
Sobrepeso & 64 & $17,2 \%(10,0-28,2)$ & \\
$\quad$ Obeso & 35 & $37,1 \%(23,2-53,7)$ & \\
\hline
\end{tabular}

IC 95\%: intervalo de confiança de 95\%; p: significância estatística a partir do teste de quiquadrado. 
Tabela 2. Agregação dos fatores de risco para SM em adolescentes estratificados pelo sexo e estado nutricional

\begin{tabular}{|c|c|c|c|c|}
\hline & \multicolumn{4}{|c|}{ Prevalência dos fatores agregados para SM (IC 95\%) } \\
\hline & 1 fator de risco ou + & 2 fatores de risco ou + & 3 fatores de risco ou + & 4 fatores de risco ou + \\
\hline Total & $51,5 \%(47,5 \%-55,6 \%)$ & $21,5 \%(18,3 \%-25 \%)$ & $6,7 \%(4,9 \%-9 \%)$ & $0,9 \%(0,4 \%-2 \%)$ \\
\hline \multicolumn{5}{|l|}{ Sexo } \\
\hline Masculino & $55,7 \%(50 \%-61,3 \%)$ & $25,8 \%(21 \%-31,1 \%)$ & $9,4 \%(6,5 \%-13,3 \%)$ & $1 \%(0,4 \%-3 \%)$ \\
\hline Feminino & $47,5 \%(41,8 \%-53,1 \%$ & $17,3 \%(13,4 \%-22 \%)$ & $4,1 \%(2,3 \%-6,8 \%)$ & $0,7 \%(0,2 \%-2,4 \%)$ \\
\hline \multicolumn{5}{|c|}{ Estado nutricional } \\
\hline Baixo peso & $46,7 \%(30,2 \%-63,9 \%)$ & $13,3 \%(5,3 \%-30 \%)$ & --- & --- \\
\hline Normal & $46,5 \%(42 \%-51,2 \%)$ & $16,2 \%(13,1 \%-20 \%)$ & $3,3 \%(2 \%-5,4 \%)$ & --- \\
\hline Sobrepeso & $64,1 \%(51,8 \%-74,7 \%)$ & $34,4 \%(24 \%-46,6 \%)$ & $17,2 \%(10 \%-28,2 \%)$ & $1,6 \%(0,3 \%-8,3 \%)$ \\
\hline Obeso & $100 \%(90 \%-100 \%)$ & $74,3 \%(57,9 \%-85,8 \%)$ & $37,1 \%(23,2 \%-53,7 \%)$ & $11,4 \%(4,5 \%-25,9 \%)$ \\
\hline
\end{tabular}

IC 95\%: intervalo de confiança de 95\%; p: significância estatística a partir do teste de qui-quadrado.

\section{DISCUSSÃO}

Tendo em vista que a SM é forte preditora de mortalidade cardiovascular, cada vez mais estudos têm buscado destacar as características associadas a sua etiologia em indivíduos de todas as idades.

No Brasil, para o nosso conhecimento, até o momento, ainda há poucos estudos investigando a prevalência de SM em amostras representativas de adolescentes. Grande parte dos estudos conduzidos nessa área se concentrou em questões isoladas, como perfil lipídico proteico plasmático $(12,13)$, hipertensão arterial (14) ou obesidade (15), considerando os fatores de risco separadamente, sem levar em conta a importância de se avaliar a agregação destes no indivíduo.

Recentemente, Moraes e cols. (16) publicaram uma revisão sistemática sobre a prevalência de $\mathrm{SM}$ em adolescentes e, de acordo com o levantamento realizado, os autores identificaram que o Brasil carece de estudos que estimem a prevalência de SM em adolescentes, uma vez que nenhum estudo foi encontrado.

Pouco tempo após essa revisão, Rodrigues e cols. (17) divulgaram informações sobre o diagnóstico de SM em 380 adolescentes de 10 a 14 anos de idade da cidade de Vitória, ES, sendo a prevalência geral de 1,3\% (meninos $1,1 \%$ e meninas $1,5 \%$ ), muito abaixo dos valores encontrados no presente estudo, fator esse explicado pelo critério adotado pelos autores para o diagnóstico de SM.

Dados do presente estudo demonstraram que a prevalência geral de SM em adolescentes paranaenses foi de $6,7 \%$, no entanto, nos adolescentes com excesso de peso, essa prevalência aumentou para $17,2 \%$ nos indivíduos com sobrepeso e $37,1 \%$ nos obesos. Como não há estudos representativos com adolescentes brasi- leiros sobre SM, nossos achados constituem referência importante para que outros estudos sejam conduzidos.

Dados publicados previamente na literatura internacional reportaram taxas de prevalência de SM entre $2 \%$ e 9\% em adolescentes, consistentes com nossos achados. Cook e cols. (4) encontraram prevalência global da SM em 4,2\% dos adolescentes. Quando os autores avaliaram a prevalência da SM estratificados pelo estado nutricional, esses valores subiram para $28,7 \%$ nos adolescentes obesos (IMC $\geq$ percentil $95^{\circ}$ ). Da mesma forma, Cruz e cols. (18) revelaram prevalência de SM em $38,1 \%$ dos jovens latino-americanos obesos participantes da pesquisa.

No estudo de Ferranti e cols. (19), a prevalência de SM foi de 9,2\% nos indivíduos com peso normal e $31,2 \%$ nos obesos. Weiss e cols. (20) encontraram prevalência de SM em 38,7\% dos adolescentes moderadamente obesos e $49,7 \%$ nos severamente obesos.

Recentemente, Cook e cols. (21) avaliaram as taxas de prevalência da SM (NHANES 1999-2002) e verificaram que a síndrome acometeu $44 \%$ dos adolescentes obesos, bem acima dos 37,1\% encontrados no presente estudo.

Essa associação direta do excesso de peso com o diagnóstico de SM demonstrada nos estudos prévios causa grande preocupação, uma vez que dados da última pesquisa de orçamento familiar realizada pelo Instituto Brasileiro de Geografia e Estatística (IBGE) em 2002/03 (22) evidenciaram aumento da obesidade e redução dos índices de desnutrição em brasileiros.

Além disso, estudos longitudinais demonstram que múltiplos fatores de risco cardiovascular persistem da infância até a idade adulta em $25 \%$ a $60 \%$ dos casos. Pesquisas que acompanharam adolescentes até a idade adulta observaram que mais de $50 \%$ dos indivíduos que 
apresentavam excesso de peso, quando adolescentes, permaneceram nesse estado quando adultos $(23,24)$.

Assim, estratégias de intervenção devem ser pensadas já precocemente objetivando a saúde a longo prazo. Nesse sentido, mudanças nos hábitos alimentares e adoção de um estilo de vida fisicamente mais ativo são ferramentas de grande valia. Pesquisas que avaliaram a associação da SM com os níveis de atividade física observaram que a atividade física está inversamente associada ao risco metabólico em adultos (25) e crianças (26), e que o sedentarismo aumenta a chance de seu desenvolvimento $(27,28)$.

Guimarães e Ciolac (29) abordaram em sua revisão da literatura sobre a importância de exercícios físicos no controle dos principais componentes da SM. Os autores concluíram que programas focados na prevenção e no tratamento da SM devem incluir componentes que melhorem o condicionamento cardiorrespiratório, a força e a resistência muscular.

Algumas questões devem ser levantadas sobre a prevalência da SM em indivíduos jovens. A primeira se refere a como diagnosticar SM em crianças e adolescentes, uma vez que os estudos descrevem valores percentuais da SM, entretanto, as comparações entre os estudos se tornam difíceis, pois, em virtude da falta de um consenso sobre o diagnóstico da SM na população pediátrica, se aplicam definições e pontos de corte diferentes.

Outro fato a ser considerado é a classificação do estado nutricional pelo IMC, o qual pode superestimar a prevalência de SM, uma vez que a obesidade abdominal medida pela circunferência da cintura está fortemente correlacionada com a obesidade total classificada pelo IMC.

Em resumo, nossos resultados demonstram que a prevalência de SM entre adolescentes é preocupante, principalmente nos indivíduos que apresentam excesso de peso. Novos estudos devem ser conduzidos para elevar o conhecimento sobre as características associadas ao desenvolvimento da SM em crianças e adolescentes, para que, num futuro próximo, estratégias de prevenção possam ser desenvolvidas com foco no controle do peso já no início da vida.

Declaração: os autores declaram não haver conflitos de interesse científico neste estudo.

\section{REFERÊNCIAS}

1. National Cholesterol Education Program (NCEP) Expert Panel on Detection, Evaluation, ad Treatment of High Blood Choleste- rol in Adults (Adult Treatment Panel III): Executive Summary of the Third Report of The National Cholesterol Education Program (NCEP) Expert Panel on Detection, Evaluation, ad Treatment of High Blood Cholesterol In Adults (Adult Treatment Panel III). JAMA. 2001;285:2486-97.

2. HitsumotoT, Makahashi M, LizukaT, Shirai K. Relationship between metabolic syndrome and early stage coronary atherosclerosis. $J$ AtherosclerThromb. 2007;14:294-302.

3. Haffner SM, Valdez RA, Hazuda, HP, Mitchell BD, Morales PA, Stern MP. Prospective analysis of the insulin-resistance syndrome (syndrome X). Diabetes. 19s92;41:715-22.

4. Cook S, Weitzman M, Auinger P, Nguyen M, Dietz WH. Prevalence of a metabolic syndrome phenotype in adolescents: findings from the Third National Health and Nutrition Examination Survey, 1988-1994. Arch Pediatr Adolesc Med. 2003;157:821-7.

5. Sociedade Brasileira de Cardiologia. I Diretriz Brasileira de Diagnóstico e Tratamento da Síndrome Metabólica. Arq Bras Cardiol. 2005;84:s1-s28.

6. Salaroli LB, Barbosa GC, Mill JG, Molina MCB. Prevalência de síndrome metabólica em estudo de base populacional, Vitoria, ES - Brasil. Arq Bras Endocrinol Metab. 2007;51:1143-52.

7. Chen W, Berenson GS. Metabolic syndrome: definition and prevalence in children. J Pediatr (Rio J). 2007;83:1-3.

8. Brandão AP, Brandão AA, Berenson GS, Fuster V. Síndrome metabólica em crianças e adolescentes. Arq Bras Cardiol. 2005;85:79-81.

9. Katzmarzyk PT, Perusse L, Malina RM. Stability of indicators of the metabolic syndrome from childhood and adolescence to young adulthood: the Quebec Family Study. J Clin Epidemiol. 2001;54:190-5.

10. National Center for Health Statistics. CDC Growth Charts: United States. 2000;4:314.

11. National High Blood Pressure Education Program Working Group High Blood Pressure in Children and Adolescents. The fourth report on the diagnosis, evaluation, and treatment of high blood pressure in children and adolescents. Pediatrics. 2004;114:555-76.

12. Guedes DP, Guedes JERP, Barbosa DS, Oliveira JA, Stanganelli ICR. Fatores de risco cardiovasculares em adolescentes: indicadores biológicos e comportamentais. Arq Bras Cardiol. 2006;86:439-50.

13. Ribeiro RQC, Lotufo PA, Lamounier JA, Oliveira RG, Soares JF, Botter DA. Fatores adicionais de risco cardiovascular associados ao excesso de peso em crianças e adolescentes. 0 estudo do coração de Belo Horizonte. Arq Bras Cardiol. 2006;86:408-8.

14. Stabelini Neto A, Mascarenhas LPG, Vasconcelos IQA, Bozza R, Ulbrich AZ, Campos W. High blood pressure in the adolescence: relationship with the cardiorespiratory fitness, $\mathrm{BMI}$ and waist circumference. Rev Bras Hipertens. 2008;15:59-64.

15. Mascarenhas LPG, Salgueirosa FM, Nunes GF, Martins PA, Stabelini Neto $A$, Campos W. Relação entre diferentes índices de atividade física e preditores de adiposidade em adolescentes de ambos os sexos. Rev Bras Med Esporte. 2005;11:214-8.

16. Moraes ACF, Fulaz CS, Oliveira ERN, Reicher FF. Prevalência de síndrome metabólica em adolescentes: uma revisão sistemática. Cad Saúde Pública. 2009;25:1195-202.

17. Rodrigues AB, Perez AJ, Pires JGP, Carletti I, Araújo MTM, Moyses MR, et al. Fatores de risco cardiovasculares, suas associações e presença de síndrome metabólica em adolescentes. J Pediatr (Rio J). 2009;85:55-60.

18. Cruz ML, Weigensberg MJ, Huang TTK, Ball G, Shaibi GQ, Goran MI.The metabolic syndrome in overweight Hispanic youth and the role of insulin sensivity. J Clin Endocrinol Metab. 2004;89:108-13.

19. Ferranti SD, Gauvreau K, Ludwig DS, Neufeld EJ, Newburger JW, Rifai N. Prevalence of the metabolic syndrome in American adolescents: findings from the Third National Health and Nutrition Examination Survey. Circulation. 2004;110:2494-7. 
20. Weiss R, Dziura J, Burgert TS, Tamborlane WV, Taksali SE, Yechel $\mathrm{CW}$, et al. Obesity and the metabolic syndrome in children and adolescents. N Engl J Med. 2004;350:2362-74.

21. Cook $S$, Auinger $P, L i C$, Ford E. Metabolic syndrome rates in United States adolescents, from the National Health and Nutrinion Examination Survey, 1999-2002. J Pediatr. 2008;152:165-70.

22. IBGE. Pesquisa de orçamentos familiares. Análise da disponibilidade domiciliar de alimentos e do estado nutricional no Brasil. Rio de Janeiro: IBGE; 2004.

23. Must A, Jacques, PF, Dallal GE, Bajema CJ, Dietz WH. Long-term morbity and mortality of overweight adolescents: a follow-up of the Harvard Growth Study 1922 to 1935. N Engl J Med. 1992;327:1350-5.

24. Srinivasan SR, Bao W, Wattigney WA, Berenson GS. Adolescents overweights associated with adult overweight and related multiple cardiovascular risk factors: The Bogalusa Study. Metabolism. 1996;45:235-40.
25. Franks PW, Ekelud U, Brage S, Wong M, Wareham NJ. Does the association of habitual physical activity with the metabolic syndrome differ by level of cariorespiratory fitness? Diabetes Care. 2004;27:1187-93.

26. Brage S, Wedderkopp N, Ekelund U, Franks PW, Wareham NJ, Anderesen LB, et al. Features of the metabolic syndrome are associated with objectively measured physical activity ande fitness in Danish children. Diabetes Care. 2004;27:2141-8.

27. Ford ES, Kohl HW, Mokdad AH, Ajani U. Sedentary behavior, physical activity, and the metabolic syndrome among U.S. adults. Obesity Research. 2005;13:608-14.

28. Laaksonen DE, Lakka HM, Salonen JT, Niskanen LK, Rauramaa R, LakkaTA. Diabetes Care. 2002;25:1612-8.

29. Guimarães GV, Ciolac EG. Síndrome metabólica: abordagem do educador físico. Rev Soc Cardiol Estado de São Paulo 2004;4:659-70. 